\title{
Erratum: Gravitational Waves from Quasicircular Black-Hole Binaries in Dynamical Chern-Simons Gravity [Phys. Rev. Lett. 109, 251105 (2012)]
}

Kent Yagi, Nicolás Yunes, and Takahiro Tanaka

(Received 25 January 2016; revised manuscript received 28 March 2016; published 21 April 2016)

DOI: 10.1103/PhysRevLett.116.169902

In the Letter, we constructed gravitational waves emitted from compact binaries in dynamical Chern-Simons (dCS) gravity by combining both conservative and dissipative corrections. We here correct some errors that propagated into these corrections due to mistakes in our effective treatment of the scalar-field-evolution equation.

Regarding conservative corrections, the dipole-dipole interaction energy for the scalar field was derived in Eq. (5) by analogy with electromagnetism [in the text below Eq. (5), "electromagnetically charged BHs" should be replaced by "neutron stars with magnetic dipole moments"]. This was later proven in [1] by integrating out the interaction Lagrangian, which was taken to be the kinetic term for the scalar field, using the scalar-field solution. However, such a kinetic term derives only from the left-hand side of the evolution equation for the scalar field, i.e.,

$$
\square \vartheta=J,
$$

where $J$ is the effective source term of the scalar-field equation [2]. In order to obtain the correct interaction Lagrangian, one needs to also include the source term that reproduces the right-hand side of the above evolution equation, whose Lagrangian density is given by $-\vartheta J$. Accounting for such an additional term, the sign of the interaction Lagrangian now flips. This means that the signs of Eq. (5) and the first lines in Eqs. (6) and (7) need to be reversed; i.e., these equations become

$$
\begin{gathered}
E_{\mathrm{DD}}=-\frac{25}{256} \zeta \frac{m^{4}}{r_{12}^{3}} \chi_{1} \chi_{2}\left[\left(\hat{\boldsymbol{S}}_{1} \cdot \hat{\boldsymbol{S}}_{2}\right)-3\left(\boldsymbol{n}_{12} \cdot \hat{\boldsymbol{S}}_{1}\right)\left(\boldsymbol{n}_{12} \cdot \hat{\boldsymbol{S}}_{2}\right)\right], \\
\delta C_{r} \equiv \frac{25}{512} \zeta \frac{\chi_{1} \chi_{2}}{\eta}\left[\left(\hat{\boldsymbol{S}}_{1} \cdot \hat{\boldsymbol{S}}_{2}\right)-3\left\langle\left(\boldsymbol{n}_{12} \cdot \hat{\boldsymbol{S}}_{1}\right)\left(\boldsymbol{n}_{12} \cdot \hat{\boldsymbol{S}}_{2}\right)\right\rangle_{\omega}\right]-\frac{201}{3584} \zeta \frac{m^{2}}{m_{1}^{2}} \chi_{1}^{2}\left[1-3\left\langle\left(\boldsymbol{n}_{12} \cdot \hat{\boldsymbol{S}}_{1}\right)^{2}\right\rangle_{\omega}\right]+(1 \leftrightarrow 2),
\end{gathered}
$$

and

$$
\delta C_{E} \equiv-\frac{25}{256} \zeta \frac{\chi_{1} \chi_{2}}{\eta}\left[\left(\hat{\boldsymbol{S}}_{1} \cdot \hat{\boldsymbol{S}}_{2}\right)-3\left\langle\left(\boldsymbol{n}_{12} \cdot \hat{\boldsymbol{S}}_{1}\right)\left(\boldsymbol{n}_{12} \cdot \hat{\boldsymbol{S}}_{2}\right)\right\rangle_{\omega}\right]+\frac{201}{1792} \zeta \frac{m^{2}}{m_{1}^{2}} \chi_{1}^{2}\left[1-3\left\langle\left(\boldsymbol{n}_{12} \cdot \hat{\boldsymbol{S}}_{1}\right)^{2}\right\rangle_{\omega}\right]+(1 \leftrightarrow 2),
$$

respectively.

Regarding dissipative corrections, we reported in Ref. [2] that both gravitational and scalar energy flux modify the binary evolution at second post-Newtonian (PN) order. We recently realized that we did not consider some nonlinear terms in the perturbed scalar and metric field equations that also contribute at 2PN order. Taking these into account, we found that the corrections to the gravitational energy flux now become of 3PN order, while the scalar energy flux introduces a new 2PN effect in the binary evolution. These corrections are explained in more detail in [3]. This modifies Eq. (8) in the Letter to

$$
\delta C_{\mathcal{F}} \equiv \frac{25}{19776} \zeta \frac{1}{\eta^{2}}\left[\Delta^{2}+167\left\langle\left(\Delta \cdot \hat{\boldsymbol{v}}_{12}\right)^{2}\right\rangle_{\omega}\right]
$$

Notice that only the coefficient of the second term is modified.

Combining these corrected conservative and dissipative effects, the new dCS correction to the gravitational waveform phase [Eq. (9) in the Letter] is

$$
\delta C=\frac{330845}{1107456} \zeta \frac{m^{2}}{m_{1}^{2}} \chi_{1}^{2}\left[1-\frac{190107}{66169}\left(\hat{\boldsymbol{S}}_{1} \cdot \hat{\boldsymbol{L}}\right)^{2}\right]-\frac{41525}{158208} \zeta \frac{\chi_{1} \chi_{2}}{\eta}\left[\left(\hat{\boldsymbol{S}}_{1} \cdot \hat{\boldsymbol{S}}_{2}\right)-\frac{4743}{1661}\left(\hat{\boldsymbol{S}}_{1} \cdot \hat{\boldsymbol{L}}\right)(\hat{\boldsymbol{S}} 2 \cdot \hat{\boldsymbol{L}})\right]+(1 \leftrightarrow 2)
$$



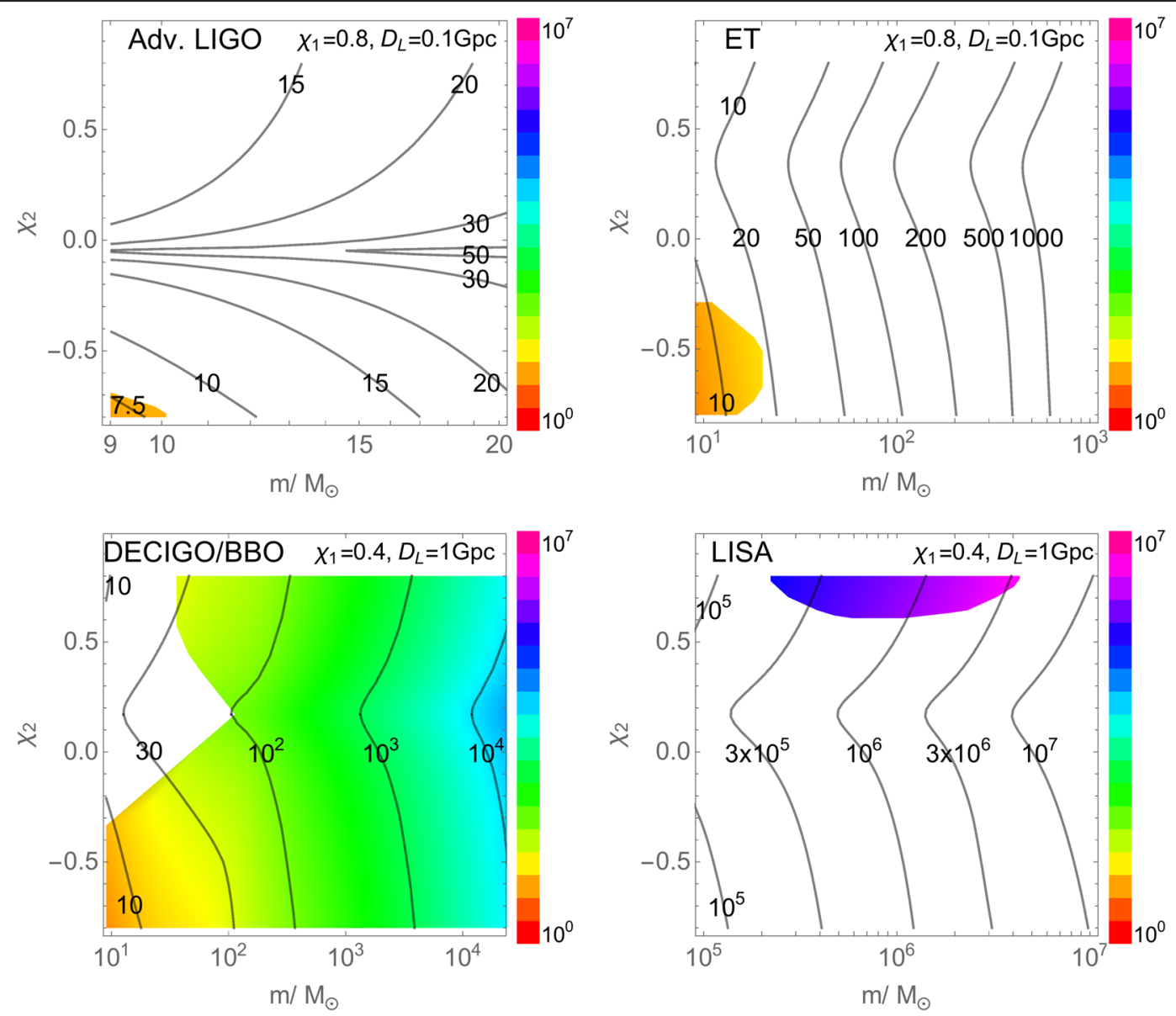

FIG. 1. Projected $1 \sigma$ constraints on $\xi^{1 / 4}$ in $\mathrm{km}$ with the second-generation ground-based detectors (top left), ET (top right), DECIGO/ BBO (bottom left), and LISA (bottom right) for black-hole binaries with $m_{1} / m_{2}=2$. The fixed values for $\chi_{1}$ and $D_{L}$ are shown at the top right of each panel. The constraints using the second-generation ground-based detectors have been obtained by assuming that the spins are known a priori. This roughly models projected constraints with precessing black-hole binary observations and may be correct within an error of $30 \%$. For other detectors, binaries are assumed to be spin aligned (or antialigned). The colored contours show the regions of parameter space where the constraints on $\xi^{1 / 4}$ also satisfy $\Delta \zeta^{\prime}<1$; thus, the small coupling approximation is satisfied at the fiducial luminosity distances chosen.

Equation (4) affects only the second and third lines in the above equation, while Eq. (5) modifies all the coefficients in Eq. (6). In the Letter, we mainly worked on spin-aligned binaries. In such cases, terms proportional to $\left\langle\left(\boldsymbol{n}_{12} \cdot \hat{\boldsymbol{S}}_{1}\right)\left(\boldsymbol{n}_{12} \cdot \hat{\boldsymbol{S}}_{2}\right)\right\rangle_{\omega}$ in Eq. (4) and $\left\langle\left(\boldsymbol{\Delta} \cdot \hat{\boldsymbol{v}}_{12}\right)^{2}\right\rangle_{\omega}$ in Eq. (5) vanish; hence, the only modification from the Letter enters in terms proportional to $\hat{\boldsymbol{S}}_{1} \cdot \hat{\boldsymbol{S}}_{2}$ in Eqs. (4) and (6). Below, we repeat the calculation made in the Letter and study how the modifications presented in Eq. (6) affect the results.

We have carried out a Fisher analysis with the corrected gravitational waveform under the same assumptions as in the Letter. The top left panel of Fig. 1 presents the corrected bound on $\xi^{1 / 4}$ with second-generation ground-based detectors, such as Advanced LIGO. Observe that the region in the $m-\chi_{2}$ plane (with $\Delta \zeta^{\prime}<1$ ) where the small coupling approximation is satisfied shrinks significantly from that shown in the top left panel of Fig. 2 in the Letter. The dCS correction to the phase [Eq. (6)] now vanishes at $\chi_{2} \sim 0.066$. We also carried out a Fisher analysis for precessing binaries following the Letter. We used the same assumptions and set the fiducial luminosity distance to $D_{L}=0.1 \mathrm{Gpc}\left(D_{L}=0.11 \mathrm{Gpc}\right.$ in the Letter is a typographical error). We found that $\zeta^{\prime}<53.3$ and $\xi^{1 / 4}<40.0 \mathrm{~km}$. On the other hand, if one assumes that one knows the spins a priori, one finds $\zeta^{\prime}<16.7$ and $\xi^{1 / 4}<29.8 \mathrm{~km}$. Therefore, the results in Fig. 1 may overestimate the constraints on $\zeta^{\prime}$ by a factor of $\sim 3$ and the constraints on $\xi^{1 / 4}$ in Fig. 1 should still be accurate to roughly $30 \%$, as claimed in the Letter.

The top right panel of Fig. 1 presents the result with ET. Observe that the colored region now appears in the negative $\chi_{2}$ region instead of the positive one, which was the case in the Letter. The bottom left panel shows the result with DECIGO/ $\mathrm{BBO}$, which is not much affected. The bottom right panel shows the result with LISA, where the colored region now shrinks 
compared to that in the Letter. Overall, our main conclusion - that future gravitational-wave observations may allow us to place strong constraints on dCS gravity-is unaffected.

A new Fisher analysis using the corrected equations and a zero-detuned configuration (instead of the broadband configuration used in the Letter) for the spectral noise density curve of Advanced LIGO has been performed by the authors. The new analysis restores the significance of the results in the Letter, leading to $1 \sigma$ contours and a colored region in the $m-\chi_{2}$ plane that are similar to the original ones.

[1] K. Yagi, L. C. Stein, N. Yunes, and T. Tanaka, Phys. Rev. D 87, 084058 (2013).

[2] K. Yagi, L. C. Stein, N. Yunes, and T. Tanaka, Phys. Rev. D 85, 064022 (2012).

[3] K. Yagi, L. C. Stein, N. Yunes, and T. Tanaka, Phys. Rev. D 93, 029902(E) (2016). 